\title{
Defining Quantum Artificial Intelligence (Q.A.I)
}

\author{
MD. Sadique Shaikh*
}

ISTM Mumbai, M.S, India

*Corresponding author: MD. Sadique Shaikh, ISTM Mumbai, India

\section{Abstract}

As we knew already Artificial Intelligence (A.I) is mimic of Natural Intelligence (N.I) of Human Brain on Silicon Chips having all intelligence processing as similar and possible for human brain with self-decisions, self-controls, self-programs, self-thinking with time management. Due to the advancement in A.I each day Humankind lives becoming comfortable, fast, smart and successful and those things which are seems to be impossible started to become possible from past to present in all walks of life. Now refocus our attention on Natural Intelligence (God-made) of mankind and other intelligent species on planet earth. When we genuinely observed we can see the biggest "Commonness" in the pattern and structure of Universe, Superclusters, Thunders, Veins, Arteries, Roots, Branches, Seas and "Neurons-Schemas" of human brain and that is the point of rethinking for new dimensions exploration in Natural Intelligence (God-made) to apply with reengineering and modification to mimic Artificial Intelligence (Man-made) on the basis of those findings which I coined as "Quantum Artificial Intelligence (Q.A.I)".
\end{abstract}

Keywords: Natural Intelligence (N.I), Artificial Intelligence (A.I), Quantum Natural Intelligence (Q.N.I), Quantum Artificial Intelligence (Q.A.I).

\section{Introduction}

(Figure 1) As I mentioned already analogically pattern of our Universe, Superclusters and Human Brain Neural-Schemas are the same. Hence not only signals transmissions and receptions for Intelligence processing are the same but also Intelligence signals nature (format) are the same which is Quanta (Light). Entire Universe is the mixture of light and dark energies and matter as its building blocks, therefore all the living/non-living creatures, objects, entites, elements, occurrences, appearances, illusions either made from or born using same building blocks lights with various frequencies for isolations and that's why human brain too. On the basis of available some proved research write-ups and with my perspective Universe and Every Human Brain directly connected and link up/down with light frequency and Quantum Mechanics also engaged to prove it. Our Thoughts are the Things, our perception and formulation to life and Universe also because of same happened, thus what all we feel physical in actual "Virtual" and are Quantum images, frames, pictures or illusions of our thoughts frequency in front appearance of our eyes to develop, structure and enhance our intelligence called wisdom too. Hence Natural Intelligence is made up of light energies forms and various tuned frequencies (according to Stings theory) called "Quantum Natural Intelligence (Q.N.I)" and when after full flagged understanding and clearance in Q.N.I we will be able to mimic it artificially called "Quantum Artificial Intelligence (Q.A.I)". the concept herein instead of Silicon Chips or Electronics Artificial
Intelligence, A.I engineering using lights forms, light signals circuits or Quantum Circuits which are just a right and precise combinations of different wavelengths and light frequencies (Quanta/Photons) with behaving as wave (light data buses) and particles (Signals) for light data buses and signals to formulate processing logics and build-up Artificial Intelligence using light. In mankind in future develop such a light which seems to be just radiation but in actual complicated light engineering using light waves and particles (Quanta) in billions of Quanta's and acting as complete Quantum Artificial Intelligence (Q.A.I) based robotic assembly to win the world, Universe and Multiverse.

\section{Conclusion}

If in near future scientist and researchers able to understand what I am tried to share as biggest fact of the Universe and the Human Brain that, all brains directly connected with light frequency with entire Universe and the structure of Universe, Superclusters and Human Brain Neural-Schemas are the same as well as intelligence processing also same using the concepts and principles of Quantum Physics and Quantum Mechanics. Therefore, on this ground Human Brain is Quantum Natural Intelligence (Q.N.I) and all formulation in brain of life and Universe due to the light intelligence. After understanding this phenomenon of Q.N.I scientist and engineers can move for the engineering of "Quantum 
Artificial Intelligence (Q.A.I) which would be just appearances of light bundles and bunches with various color lights with different wavelength confined at single spot but in actual it would be space robots, space craft, and transportation sources and so on. Hence this intelligent light form can send/transmit with the speed of light for instant time and space travels in Universe to explore other planets, galaxies, Superclusters, stars and also to prove the concept of "Multiverse (Parallel Universes)". Human Intelligence as Quantum Natural Intelligence (Q.N.I) and its mimic Artificial Intelligence as Quantum Artificial Intelligence (Q.A.I) will become exactly same, therefore direct connection between mankind and all robots will be possible without encodings, decoding's and interfaces for communication with intelligent signals processing, conversions, translation and actuations. Hence Q.A.I is nothing but Ultra Artificial Intelligence light form (light A.I based robotics) and might be such a several Q.A.I robots, space craft, objects and Alien lives already exist in entire Universe and Multiverse and we are surrounded with them, and that what we just consider or think as light or radiation but which is not.

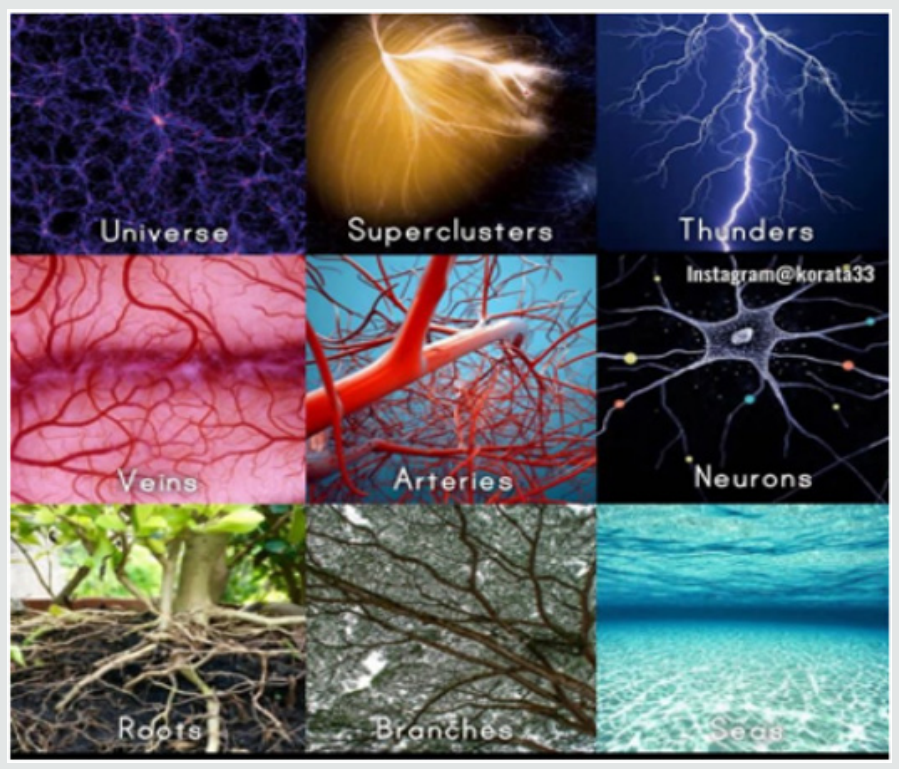

Figure 1.

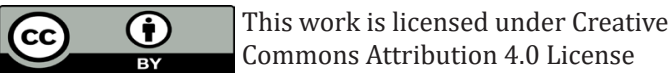

To Submit Your Article Click Here:

Submit Article

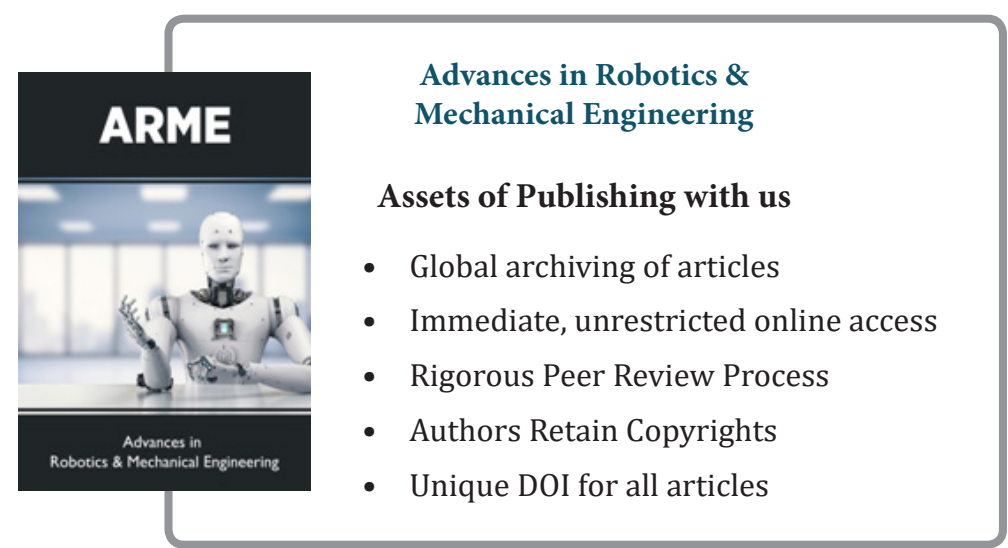

